\title{
Uncertainty estimation in railway track life cycle cost: a case study from Swedish National Rail Administration
}

\author{
A P Patra*, P Söderholm, and U Kumar \\ Luleå Railway Research Center, Division of Operation and Maintenance Engineering, Luleå University of Technology, \\ Luleå, Norbotten, Sweden
}

The manuscript was received on 5 May 2008 and was accepted after revision for publication on 17 October 2008.

DOI: 10.1243/09544097JRRT235

\begin{abstract}
Life cycle cost (LCC) is used as a cost-effective decision support for maintenance of railway track infrastructure. However, a fair degree of uncertainty associated with the estimation of LCC is due to the statistical characteristics of reliability and maintainability parameters. This paper presents a methodology for estimation of uncertainty linked with LCC, by a combination of design of experiment and Monte Carlo simulation. The proposed methodology is illustrated by a case study of Banverket (Swedish National Rail Administration). The paper also includes developed maintenance cost models for track.
\end{abstract}

Keywords: reliability and maintainability, life cycle cost, railway track, design of experiment, uncertainty

\section{INTRODUCTION}

Life cycle cost (LCC) takes into account all costs associated with the life time of the system, such as operating costs, maintenance costs, energy costs, and taxes apart from capital costs. For many complex assets, the cost of maintenance plays an important role in the LCC analysis, especially for assets like track infrastructure, where the operation and maintenance phase comprises a major share of the system's life cycle. However, though most infrastructure managers today consider all the costs incurred by the system from conceptual design to disposal in their LCC calculations, there are still some issues associated with the correctness of these calculations. Some important issues are related to uncertainties in the LCC calculations.

Figure 1 illustrates two different levels of uncertainties associated with LCC of track infrastructure. Level I uncertainty is costs due to penalties imposed by traffic operators on the infrastructure manager due to such factors as train delay, traffic disruption, or derailment. These anomalies can be caused by planned or unplanned maintenance actions, but also by lack

\footnotetext{
*Corresponding author: Division of Operation and Maintenance Engineering, Luleå University of Technology, Luleå, Norbotten 97187, Sweden.email:ambika.patra@ltu.se
}

of necessary maintenance. Hence, the resulting costs are related to decisions about maintenance actions and can be estimated by probabilistic assessment of train delay, derailment, or traffic disruptions considering the technical and operational characteristics of the track, as well as the maintenance actions. Level I uncertainty can also be viewed as belonging to the external risk of the LCC analysis, where the costs should be included to make the LCC analysis more effective. However, there is also the level II uncertainty, which is the internal risk associated with LCC. The level II uncertainty pertains to the variable contribution to total LCC originating from the uncertainty in reliability and maintainability (R\&M) parameters. However, the R\&M parameters also indirectly impact the level I uncertainty. As conventional LCC analysis only considers point estimates of R\&M parameters, it leads to an incorrect estimate of the LCC. To get a more correct estimate of the LCC, it is essential to also consider the interval estimate of the $R \& M$ parameters.

There is some research related to the stochastic nature of R\&M parameters included in LCC estimation of railway infrastructure, see e.g. [1]. However, no published research about the estimation of the uncertainty in LCC of railway infrastructure has been found. Hence, this paper aims at describing a methodology that can be used for uncertainty estimation in railway infrastructure LCC. 


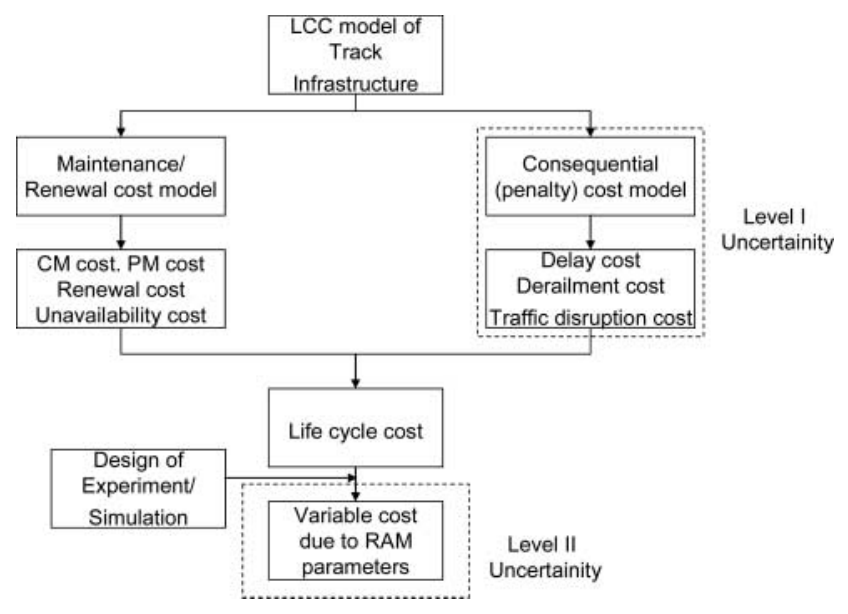

Fig. 1 Uncertainties involved with LCC modelling

\section{MAINTENANCE COST MODELLING}

LCC, which is generally modelled in the design phase, changes when the system enters into the operation and maintenance phase due to changes in stakeholder requirements, which makes the costs incurred during the operation and maintenance phase predominant. Maintenance costs of track infrastructure consist of preventive maintenance cost, renewal cost, and corrective maintenance cost (Table 1).

Maintenance costs are the most complex cost component of an asset during its life since maintenance is a long continuous process throughout the asset life. While the cost of any specified maintenance work on an asset can be comfortably estimated using engineering costing methodologies, estimating maintenance costs throughout the asset life is a much more sophisticated process. This is because the types of maintenance are dependent on many factors, of which the most important are asset deterioration rates, maintenance policy, and budget constraint. Maintenance schedules therefore need to be planned to enable the

Table 1 Track maintenance at Banverket

\begin{tabular}{|c|c|c|}
\hline $\begin{array}{l}\text { Maintenance } \\
\text { strategy }\end{array}$ & $\begin{array}{l}\text { Maintenance } \\
\text { action }\end{array}$ & $\begin{array}{l}\text { Maintenance } \\
\text { trigger }\end{array}$ \\
\hline Preventive maintenance & $\begin{array}{l}\text { Rail grinding } \\
\text { Tamping } \\
\text { Rail lubrication } \\
\text { Ballast cleaning } \\
\text { Track inspection }\end{array}$ & $\begin{array}{l}\text { Time } \\
\text { Condition } \\
\text { Time } \\
\text { Condition } \\
\text { Time }\end{array}$ \\
\hline $\begin{array}{l}\text { Renewal } \\
\text { (preventive } \\
\text { maintenance) }\end{array}$ & $\begin{array}{l}\text { Rail renewal } \\
\text { Ballast renewal } \\
\text { Sleeper renewal } \\
\text { Fasteners renewal }\end{array}$ & $\begin{array}{l}\text { Condition } \\
\text { Condition } \\
\text { Condition } \\
\text { Condition }\end{array}$ \\
\hline Corrective maintenance & Rail replacement & Failure \\
\hline
\end{tabular}

maintenance costs to be estimated. Maintenance costs of track must include:

(a) materials, equipment, and labour;

(b) condition monitoring and inspection;

(c) track possession time.

The maintenance process at Banverket (Swedish National Rail Administration) is divided into corrective and preventive maintenance, where the later is based on condition or time. The current strategy at Banverket is to minimize corrective maintenance and to change time-based maintenance to condition-based [2]. Table 1 shows the different track maintenance strategies and actions at Banverket.

In this paper, maintenance costs associated with track have been estimated separately for different curve radii as different curve radii experience different failure probabilities and magnitudes. In this paper, maintenance cost models have been developed with respect to the type of maintenance intervention summarized in Table 1. The maintenance costs have been determined as per the maintenance policy followed at Banverket. The track has been divided into different sets of curve radii $(K)$, i.e. $0-300 \mathrm{~m}(K=1), 300-450 \mathrm{~m}$ $(K=2), 450-600 \mathrm{~m}(K=3)$, and so on. Curves with radius more than $2000 \mathrm{~m}$ have been considered as tangent track. The segmentations of the track have been done as per of availability of the track failure data.

A few things must be considered while performing the segmentations of a track section. The segmentation of the track must be done for a specific track section and should not be generalized. The segmentation of the track must done as per (a) the number of each individual curve existing in a track section and (b) the number of track failures occurring in each type of curve over a period of time. For example, if there are few curves of curve radii between 700 and $1000 \mathrm{~m}$, it is safe to take $700-1000 \mathrm{~m}$ as one segment, whereas if there are a lot of curves existing of curve radii between 500 and $600 \mathrm{~m}$ the $500-600 \mathrm{~m}$ must be defined as a track segment. The same logic can be applied for the number of failures in different curve radii. If the numbers of curves as well as the numbers of failures are high in a particular segment, it can be still divided into further segments. The segmentation of track section should be specific for each studied region, as described above.

Different track maintenance and renewal costs are illustrated below.

\subsection{Rail grinding cost}

Grinding is the maintenance action done on the rail to control rolling contact fatigue defects. Cost due to rail grinding primarily depends on the periodicity of grinding and the number of grinding passes and is 
given by

$$
\sum_{i=1}^{K} \sum_{j=1}^{N-1} \frac{\begin{array}{c}
\left(\left(T_{\mathrm{g}_{i}} * C_{\mathrm{L}} * L_{i} * n_{\mathrm{g}_{i}}\right)+\left(C_{\mathrm{eg}} * T_{\mathrm{g}_{i}} * L_{i} * n_{\mathrm{g}_{i}}\right)\right) \\
*\left(m / m_{\mathrm{g}_{i}}\right)
\end{array}}{(1+r)^{j}}
$$

\subsection{Track tamping cost}

Tamping is the maintenance action done on the track to correct its alignment. Cost due to track tamping depends on the interval of tamping and is given by

$$
\sum_{i=1}^{K} \sum_{j=1}^{N-1} \frac{\left(\left(T_{\mathrm{ta}_{i}} * C_{\mathrm{L}} * L_{i}\right)+\left(C_{\mathrm{eta}} * T_{\mathrm{ta}_{i}} * L_{i}\right)\right) *\left(m / m_{\mathrm{ta}_{i}}\right)}{(1+r)^{j}}
$$

\subsection{Rail lubrication cost}

Lubrication is done on the rail to control rail wear. Cost due to lubrication depends on the number of lubricators in the curves and the cost to maintain each lubricator in terms of filling, which is given by

$$
\sum_{i=1}^{K} \sum_{j=1}^{N-1} \frac{\left(T_{\mathrm{clu}} * C_{\mathrm{L}} * n_{\mathrm{l}_{i}}\right)}{(1+r)^{j}}
$$

\subsection{Ballast cleaning cost}

Ballast cleaning is the maintenance action done to eliminate trapped water inside the ballast in order to restore the track quality and stiffness. Cost due to ballast cleaning primarily depends on the periodicity of ballast cleaning and is given by

$$
\sum_{i=1}^{K} \sum_{j=1}^{N-1} \frac{\left(\left(T_{\mathrm{b}_{i}} * C_{\mathrm{L}} * L_{i}\right)+\left(C_{\mathrm{eb}} * T_{\mathrm{b}_{i}} * L_{i}\right)\right) *\left(m / m_{\mathrm{b}_{i}}\right)}{(1+r)^{j}}
$$

\subsection{Track inspection cost}

Track inspection is done to detect flaws on the track that can lead to failures. The cost due to track inspection primarily depends on the interval of track inspection and is given by

$$
\sum_{j=1}^{N-1} \frac{\left(\left(T_{\mathrm{t}} * C_{\mathrm{L}} * L\right)+\left(C_{\mathrm{et}} * T_{\mathrm{t}} * L\right)\right) *\left(m / m_{\mathrm{t}}\right)}{(1+r)^{j}}
$$

\subsection{Rail renewal cost}

Rail renewal is done when the rail deterioration reaches maintenance or safety limits. The cost due to rail renewal is given by

$$
\sum_{i=1}^{K} \sum_{j=1}^{N-1} \frac{\begin{array}{c}
\left(\left(C_{\mathrm{r}} * L_{i}\right)+\left(T_{\mathrm{rr}_{i}} * C_{\mathrm{L}} * L_{i}\right)+\left(C_{\mathrm{err}} * T_{\mathrm{rr}_{i}}\right.\right. \\
\left.\left.* L_{i}\right)\right) *\left(m / m_{\mathrm{rr}_{i}}\right)
\end{array}}{(1+r)^{j}}
$$

\subsection{Ballast renewal cost}

Ballast renewal is done when ballast deterioration reaches maintenance or safety limits. The cost due to ballast renewal is given by

$$
\sum_{i=1}^{K} \sum_{j=1}^{N-1} \frac{\begin{array}{c}
\left(\left(C_{\mathrm{b}} * L_{i}\right)+\left(T_{\mathrm{br}_{i}} * C_{\mathrm{L}} * L_{i}\right)+\left(C_{\mathrm{ebr}}\right.\right. \\
\left.\left.* T_{\mathrm{br}_{i}} * L_{i}\right)\right) *\left(m / m_{\mathrm{br}_{i}}\right)
\end{array}}{(1+r)^{j}}
$$

\subsection{Sleeper renewal cost}

Sleeper renewal is done when the sleeper deterioration reaches maintenance or safety limits. The cost due to sleeper renewal is given by

$$
\sum_{i=1}^{K} \sum_{j=1}^{N-1} \frac{\begin{array}{r}
\left(\left(C_{\mathrm{s}} * L_{i}\right)+\left(T_{\mathrm{sr}_{i}} * C_{\mathrm{L}} * L_{i}\right)+\left(C_{\mathrm{esr}} * T_{\mathrm{sr}_{i}} * L_{i}\right)\right) \\
*\left(m / m_{\mathrm{sr}_{i}}\right)
\end{array}}{(1+r)^{j}}
$$

\subsection{Fastener renewal cost}

Fastener renewal is done when the fastener deterioration reaches maintenance or safety limits. The cost due to fastener renewal is given by

$$
\sum_{i=1}^{K} \sum_{j=1}^{N-1} \frac{\begin{array}{l}
\left(\left(C_{\mathrm{f}} * L_{i}\right)+\left(T_{\mathrm{fr}_{i}} * C_{\mathrm{L}} * L_{i}\right)+\left(C_{\mathrm{efr}} * T_{\mathrm{fr}_{i}} * L_{i}\right)\right) \\
*\left(m / m_{\mathrm{fr}_{i}}\right)
\end{array}}{(1+r)^{j}}
$$

\subsection{Rail replacement cost}

Rail replacement is done when rail breaks occur on the track. Cost due to rail break primarily depends on the probability of rail breaks and is given by

$$
\sum_{i=1}^{K} \sum_{j=1}^{N-1} \frac{\begin{array}{c}
\left(\left(C_{\mathrm{r}} * L_{\mathrm{r}}\right)+\left(T_{\mathrm{rb}_{i}} * C_{\mathrm{L}}\right)+\left(C_{\mathrm{er}} * T_{\mathrm{rb}_{i}}\right)\right) \\
*\left(m / m_{\mathrm{rb}_{i}}\right)
\end{array}}{(1+r)^{j}}
$$




\subsection{Track downtime cost}

Downtime on the track occurs due to track possession for maintenance actions on the track. Train-free periods are usually used for planning maintenance actions, i.e. the hours between two consecutive trains. However, as the train-free periods are not long enough in most cases, this leads to train cancellations, train speed restrictions, etc., which imply penalties imposed on the infrastructure manager by the traffic operators. Preventive maintenance and renewal actions are usually planned well ahead so as not to affect the traffic. However, corrective maintenance on the track generally affects the train operation. In this case, rail breaks have been considered for corrective maintenance. Mean time to repair (MTTR) for rail break is given by

$$
\frac{\sum_{i} f_{\mathrm{rb}_{i}} * T_{\mathrm{rb}_{i}}}{\sum_{i} f_{\mathrm{rb}_{i}}}
$$

In this case, track possession time is calculated as the difference between MTTR and train-free period. Hence, the track downtime cost can be calculated by multiplying the track possession period with the penalty cost. Table 2 describes the R\&M parameters associated with track maintenance.

\section{UNCERTAINTY IN LCC}

The statistical characteristics of R\&M parameters contribute to uncertainty in LCC. The reason for this is that the times and conditions for these types of events are so complex that they cannot be predicted with a fair degree of accuracy. Therefore, it was decided to explore a methodology that combines the use of Design of Experiment (DoE) principles with Monte Carlo simulation to estimate the uncertainty involved with LCC. The area of DoE was developed in the twentieth century to increase the effectiveness and efficiency of experimentation. However, for experiments to be effective and lead to correct conclusions there are a

Table 2 R\&M parameters associated with track maintenance

\begin{tabular}{llc}
\hline Maintenance actions & \multicolumn{2}{c}{ R\&M parameters } \\
\hline Rail grinding & $T_{\mathrm{g}_{i}}$ & $m_{\mathrm{g}_{i}}$ \\
Tamping & $T_{\mathrm{ta}_{i}}$ & $m_{\mathrm{ta}_{i}}$ \\
Rail lubrication & $T_{\mathrm{lu}_{\mathrm{u}}}$ & \\
Ballast cleaning & $T_{\mathrm{b}_{i}}$ & $m_{\mathrm{b}_{i}}$ \\
Track inspection & $T_{\mathrm{t}}$ & \\
Rail renewal & $T_{\mathrm{rr}_{i}}$ & $m_{\mathrm{rr}_{i}}$ \\
Ballast renewal & $T_{\mathrm{br}_{i}}$ & $m_{\mathrm{br}_{i}}$ \\
Sleeper renewal & $T_{\mathrm{sr}_{i}}$ & $m_{\mathrm{sr}_{i}}$ \\
Fasteners renewal & $T_{\mathrm{fr}_{i}}$ & $m_{\mathrm{fr}_{i}}$ \\
Rail replacement & $T_{\mathrm{rb}_{i}}$ & $m_{\mathrm{rb}_{i}}$ \\
Downtime cost & $T_{\mathrm{rb}_{i}}$ & $f_{\mathrm{rb}_{i}}$ \\
\hline
\end{tabular}

number of requirements that should be fulfilled [3]. For example, the response must be measurable and be correlated to the purpose of the experiment. Furthermore, even though not an absolute necessity, the power of statistical operations will be greater if the response is continuous and preferably also normally distributed. The responses of this study are the point estimate for LCC of the track and its related uncertainty, which both are continuous, but not necessarily normally distributed. The following are valid for the present study.

1. The factors that are tested in the experiment are R\&M-parameters, which all are continuous and numeric. They are also measurable, controllable, and deemed important for the selected responses.

2. The factors that are not under investigation can easily be held constant, since the study is analytical and not empirical. These factors are the cost factors not directly related to R\&M. Hence, no randomization is considered necessary.

Since the study is analytical there are no major economical constraints. Hence, the design is mostly dependent upon the number of R\&M parameters that are to be investigated. In order to fulfil the purpose of this study, a two-level factorial design is considered valuable. However, in order to reduce the number of runs, a fractional factorial design is considered sufficient. The analysis is supported by the software tool STATGRAPHICS, which provides suitable tables and graphs for presentation.

The probability distribution of LCC can be found by the use of Monte Carlo simulation. A Monte Carlo simulation is effectively a random number generator that creates values for each $R \& M$ parameter. Values are chosen within specified ranges of each parameter and with a frequency proportional to the shape of probability distribution associated with each R\&M parameter. The proposed methodology helps in determining the variable costs associated in LCC estimation. These variable costs can be termed as the uncertainty in LCC estimation and are caused by the probabilistic nature of the R\&M parameters. The LCC becomes more robust when these variable costs are included. Thus, it helps the decision makers to make more effective decisions on maintenance policy by considering LCC.

\section{CASE STUDY}

The performed case study was on the iron ore line (Malmbanan) that runs from Luleå in Sweden to Narvik in Norway. The line allows 30 tonne axle load with mixed traffic. Data (Tables 3 and 4) was collected from Banverket's failure and maintenance databases (i.e. BIS, Bessy, and Ofelia) that range from 1997 to 2006 with some data being collected from reference [4]. The 
Table 3 TTF (rail break) data in MGT for curves of radius $450-600 \mathrm{~m}$

\begin{tabular}{ll}
\hline High rail & Low rail \\
\hline 400 & 325 \\
350 & 350 \\
250 & 150 \\
425 & 225 \\
300 & 275 \\
325 & 425 \\
150 & 300 \\
350 & 125 \\
150 & 150 \\
400 & 400 \\
275 & 300 \\
575 & \\
\hline
\end{tabular}

Table 4 TTR (to correct rail break) data in minutes for curves of radius $450-600 \mathrm{~m}$

\begin{tabular}{lc}
\hline High rail & Low rail \\
\hline 159 & 258 \\
120 & 154 \\
480 & 216 \\
149 & 240 \\
270 & 169 \\
547 & 75 \\
340 & 340 \\
43 & 202 \\
228 & 202 \\
202 & 216 \\
240 & 240 \\
218 & \\
\hline
\end{tabular}

study was performed on the rail replacement cost on high and low rails separately. Low rail denotes the inner rail (smaller radius) and high rail the outer rail (larger radius) in a curved track. The idea of separating high rail and low rail for cost estimation lies in the fact that they both have different failure deterioration due to quasi-static forces in the track curvatures.

The following assumptions were made after consultations with Banverket's track experts in the case study.

1. Average gross tonnage per year is assumed to be 25 million gross tonnes (MGT).

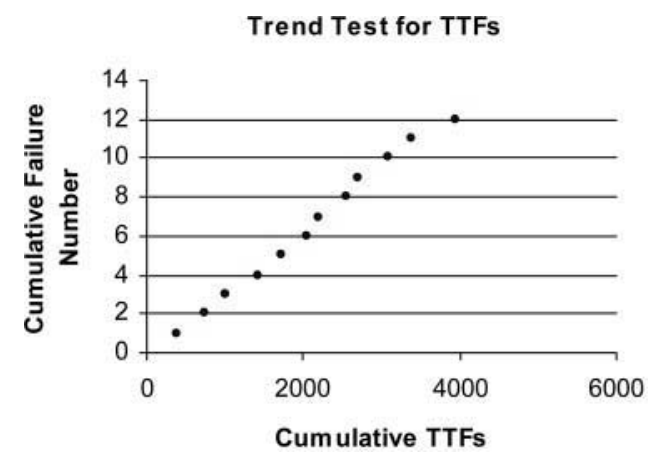

2. Life of track for LCC estimation is 600 MGT (24 years).

3. Discount rate is taken as 4 per cent.

4. Cost of BV50 rail (including neutralisation) is 1395 Swedish kronor (SEK) $/ \mathrm{m}$.

5. Average labour cost is $525 \mathrm{SEK} / \mathrm{h}$. This includes the track worker cost, track welder cost, and inspection personnel cost.

6. Welding equipment cost is $60 \mathrm{SEK} / \mathrm{h}$.

7. Average length of rail replacement due to rail break $\left(L_{\mathrm{r}}\right)$ is $8 \mathrm{~m}$.

LCC analysis was done on curves of radius 450-600 m, with cost figures given in SEK. The time to failure (TTF) and time to repair (TTR) data obtained from the Banverket data base were analysed using probability distribution models. However, before fitting any distribution models to analyse the data, the TTF and TTR data sets were verified for (independent and identically distributed (IID) random variables) assumption using graphical method (Figs 2 to 5). This is important because if data is not independent or it has trend, then probability distribution models cannot be used for analysing the data set [5]. Such data sets can be modelled by the use of other non-stationary model such as power law process model, etc [6].

Table 5 shows the probability distribution of mean time to failure (MTTF) and MTTR for both high and low rails. The analysis was supported by the software tool Weibull ++ . MTTF was estimated by considering the failure events (time period to occurrence of rail break) and suspended events (no rail break has occurred) for the particular curvatures of the track. MTTR considered here comprises of the logistic time, welding time, and inspection time necessary to repair the rail breaks. A two-sided 90 per cent confidence level was considered for determining the upper limit, mean, and lower limit of MTTF and MTTR.

Table 6 shows the LCC estimation by considering DoE principles. The high and low rails were analysed separately, but followed the same design. The applied design was a screening, full factorial, two-level design with the two experimental factors MTTF and MTTR,

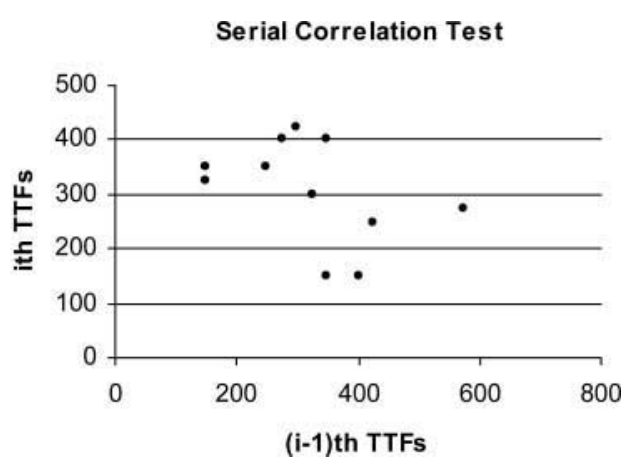

Fig. 2 Test for IID for TTFs of the high rail 

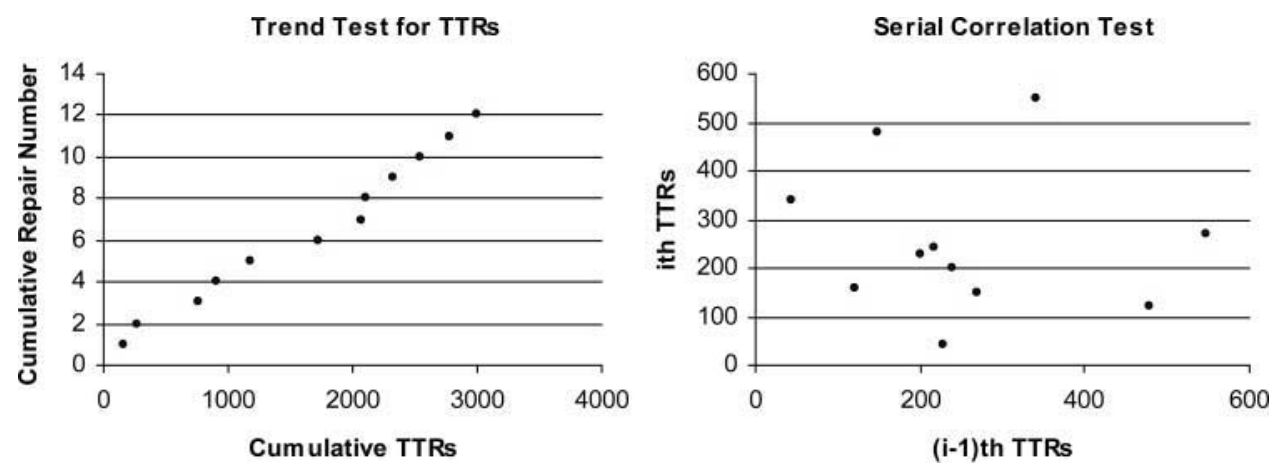

Fig. 3 Test for IID for TTRs of the high rail
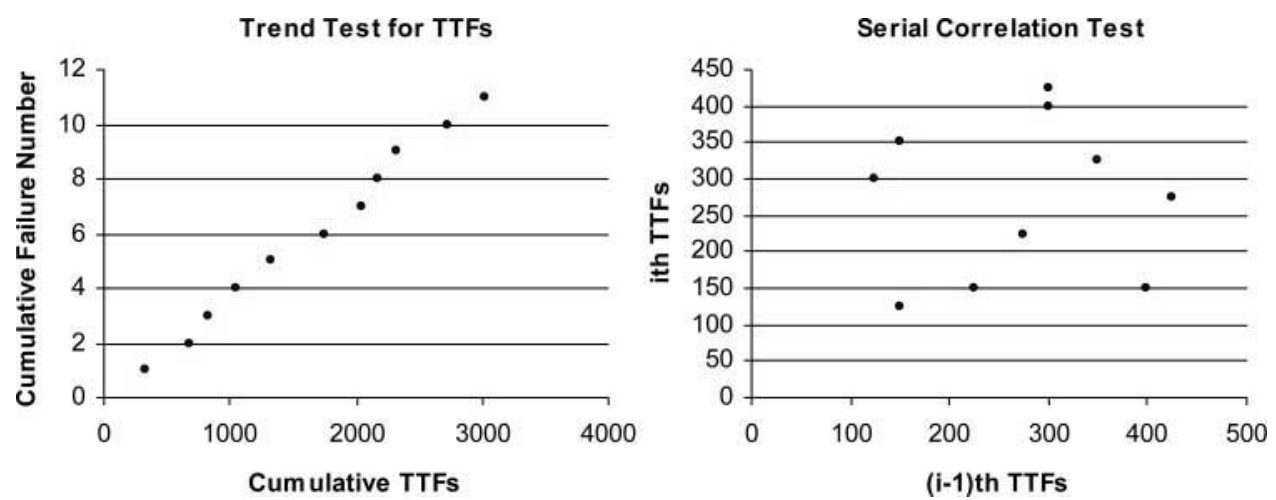

Fig. 4 Test for IID for TTFs of the low rail
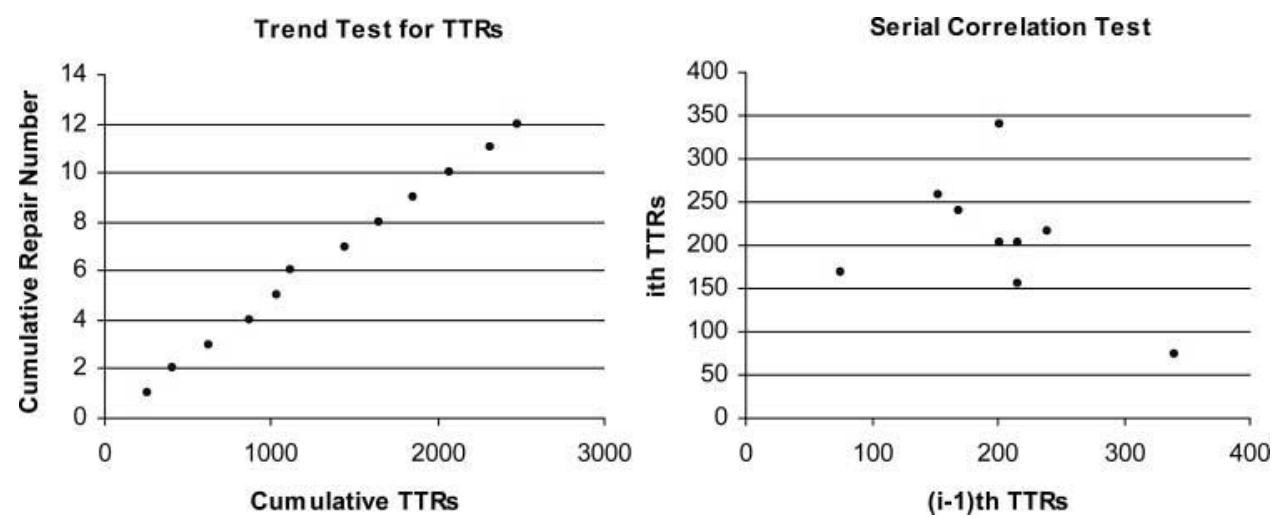

Fig. 5 Test for IID for TTRs of the low rail

i.e. a $2^{2}$-design that requires four runs. These four runs were performed ten times (i.e. ten blocks with four runs in each), resulting in a total of 40 runs for high and low rails, respectively. The high and low levels for MTTF and MTTR were selected as the upper and lower limits of their distributions. The experiment contained two responses, i.e. the point estimate and the $\log \left(s^{2}\right)$ of LCC. The rationale for analysing $\log \left(s^{2}\right)$ is described in reference [7]. The input data was generated by Monte Carlo simulations. These data were entered into equation (10) and varied according to the experimental design summarized in Table 6.
Table 6 indicates that $\log \left(\mathrm{s}^{2}\right)$ of LCC is quite stable for both high and low rails. However, changes in the levels of MTTF and MTTR do affect the variability in LCC. Since there is no interaction effect present (see Fig. 6 for example), the factors can be considered individually. An interaction between two factors means that the effects of either one cannot be judged independently. If there is an interaction between two factors, the effect of one factor on the response will depend on the setting of the other. In order to reduce the variability in LCC, one should look into the lowest value of $\log \left(s^{2}\right)$. The effects of variability in MTTF and 
Table 5 MTTF and MTTR probability distributions for high and low rails

\begin{tabular}{llll}
\hline & & High rail & Low rail \\
\hline MTTF (MGT) & Probability & Log normal $(\mu=$ & Weibull- 2 parameter \\
& distribution & $5.9933, \sigma=0.2523)$ & $(\eta=369.7161$, \\
& & & $\beta=3.5315)$ \\
& Upper limit & 482.7 & 403.5 \\
& Mean & 413.6 & 332.8 \\
& Lower limit & 354.5 & 274.4 \\
MTTR (Hours) & Probability & Weibull- 2 parameter & Normal $(\mu=3.4458$, \\
& distribution & $(\eta=4.6972$, & $\sigma=1.0296)$ \\
& Upper limit & $\beta=1.8871)$ & 3.9 \\
& Mean & 5.5 & 3.4 \\
& Lower limit & 3.1 & 2.9 \\
\hline
\end{tabular}

Table 6 LCC estimation with DoE principles

\begin{tabular}{lrrcr}
\hline Type & MTTF & MTTR & LCC (average) & $\log \left(s^{2}\right)$ \\
\hline High rail & -1 & -1 & -1050.4 & 3.4198 \\
& 1 & -1 & -940.6 & 3.4784 \\
& -1 & 1 & -1086.6 & 3.4572 \\
Low rail & 1 & 1 & -973.0 & 3.5139 \\
& -1 & -1 & -1252.1 & 3.0577 \\
& 1 & -1 & -1113.3 & 3.0128 \\
& 1 & 1 & -1288.3 & 3.0824 \\
& 1 & 1 & -1130.4 & 3.0230 \\
\hline
\end{tabular}

Interaction Plot for LOG(S^2) of LCC for Low Rail

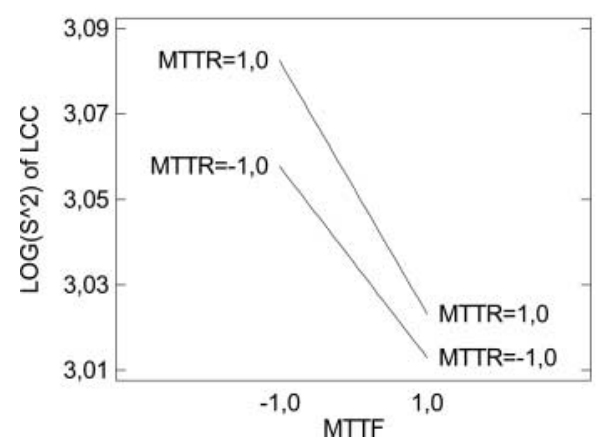

Fig. 6 Interaction plot showing variability in LCC for low rail

MTTR on the LCC of high and low rails are shown in Figs 7 and 8, respectively. The figures show the effect on LCC with increase of MTTF and MTTR values from low to high levels.

As shown in Figs 7 and 8, MTTF has a positive effect on LCC and MTTR has a negative effect. The magnitudes of the effects imply that the uncertainty in MTTF has more impact on the change in LCC than the uncertainty in MTTR. Two possible reasons for these differences in magnitudes are uncertainty levels in the parameters and given importance levels in the LCC formulation. The interaction between MTTF and MTTR is not significant in any of the cases.

Monte Carlo simulation was used to determine the probability distribution of LCC and estimate the

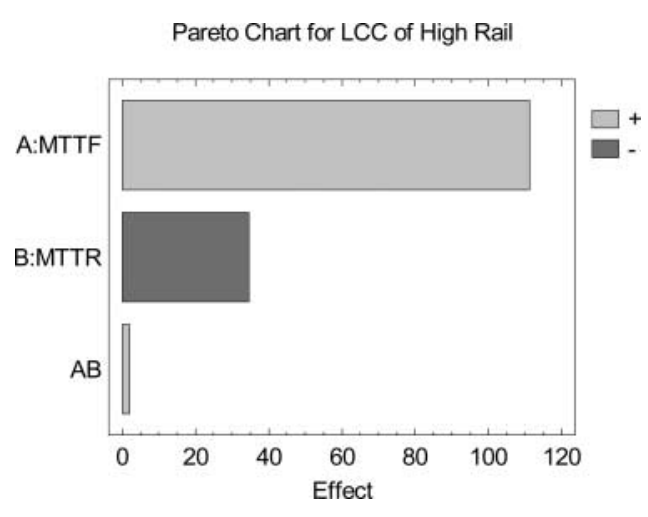

Fig. 7 Effect of MTTF and MTTR on uncertainty of LCC for high rail

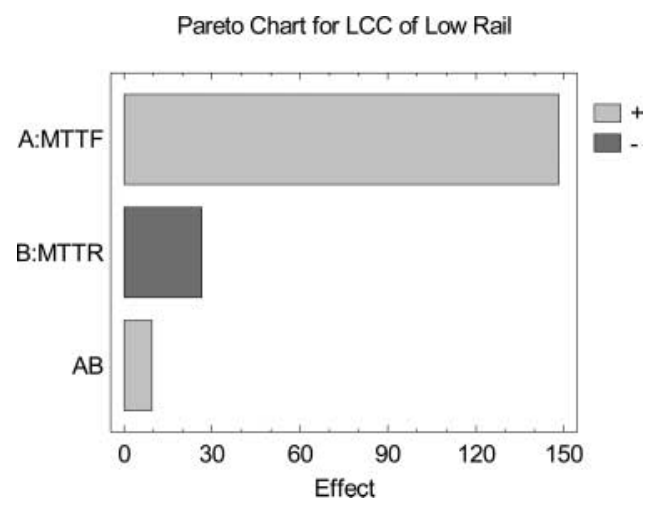

Fig. 8 Effect of MTTF and MTTR on uncertainty of LCC for low rail

associated variability cost. A two-sided 90 per cent confidence level was considered for this distribution. LCC figures were generated by combinations of upper, mean, and lower limits of MTTF and MTTR that were generated by Monte Carlo simulation. As shown in Table 7, the difference between upper and lower limits can provide the variability cost associated with LCC. The negative sign on the costs indicate that they were calculated as total present value. 
Table 7 Simulated probability distribution of LCC

\begin{tabular}{llll}
\hline & & High rail & Low rail \\
\hline LCC (SEK) & Probability & Weibull- 3 parameter & Weibull- 3 parameter \\
& distribution & Probability $(\eta=$ & $(\eta=170.7607$, \\
& $219.6026, \beta=3.0731$, & $\beta=2.2115$, \\
& Upper limit & $\gamma=815.1878)$ & $\gamma=1049.3146)$ \\
& Mean & -1024.9 & -1214.7 \\
& Lower limit & -1011.5 & -1200.5 \\
& -999.0 & -1187.6 \\
\hline
\end{tabular}

\section{DISCUSSION AND CONCLUSIONS}

LCC is being used as a tool to help in making effective maintenance decisions. However, there are various uncertainties associated with estimation of LCC. This paper presents level I and II uncertainties, out of which level II uncertainty has been dealt with. Level II uncertainty can be due to economic parameters, e.g. discounting rate, which has not been explored in this paper. The paper investigates more the uncertainties caused by technical parameters, i.e. R\&M parameters. The uncertainty in $R \& M$ parameters exist because of their probabilistic nature, which contributes to the uncertainty in LCC estimation. For better estimation of uncertainty in LCC, this paper outlines a methodology based on a combination of Monte Carlo simulation and DoE. This combination gives a possibility to identify parameters that are influential on the LCC estimation and its variability. The proposed methodology can be used to estimate the uncertainty in LCC by considering uncertainties in all parameters simultaneously, in contrast to sensitivity analysis, where the parameters are considered one by one. Hence, the methodology can contribute to other research efforts, where traditional sensitivity analyses have been performed. The simulations are used to make the deterministic LCC equations probabilistic. DoE is applied to guide how the $R \& M$ parameters should be varied in a systematic way. The paper also illustrates cost models for different maintenance and renewal actions carried out on track. The uncertainty in LCC is presented as variable costs with associated distributions. When the variable costs are added to the LCC it becomes more robust. Hence, it helps the decision-makers to make more effective decisions about maintenance policy by considering LCC.

For further research, all the developed cost models for railway track can be combined into one model. The proposed methodology can then be applied to this new cost model. However, one major challenge will be to get relevant data to use as input to this sensitivity analysis. Another challenge is to deal with the large number of runs that will result by a full two-level factorial design, since the number of runs will double with each added parameter. However, this is not any major problem since DoE principles can be applied to reduce the number of runs by using fractional factorial designs that still will give valuable information.

\section{REFERENCES}

1 Zhao, I., Chan, A. H. C., Roberts, C., and Stirling, A. B. Assessing the economic life of rail using a stochastic analysis of failures. Proc. I MechE, Part F: J. Rail and Rapid Transit, 2006, 220, 103-110.

2 Karlsson, V. An overall view of maintenance. Eur. Railw. Rev., 2005, 11 (3), 11-17.

3 Coleman, D. E. and Montgomery, D. C. A systematic approach to planning for a designed industrial experiment. Techometrics, 1993, 35, 1-27.

4 Kumar, S. A study of the rail degradation process to predict rail breaks. Licentiate Thesis, Division of Operation and Maintenance Engineering, Luleå University of Technology, Luleå, Sweden, vol. 73, 2006.

5 Ascher, H. and Feingold, H. Repairable system reliability: modelling, inference, misconceptions and their causes, 1984 (Marcel Dekker, New York).

6 Kumar, U. and Klefsjö, B. Reliability analysis of hydraulic system of LHD machines using power law process model. Reliab. Eng. Syst. Saf., 1992, 35, 217-224.

7 Bisgaard, S. and Fuller, H. T. Reducing variation with twolevel factorial experiments. Qual. Eng., 1995, 8, 373-377.

\section{APPENDIX}

\section{Notation}

$C_{\mathrm{b}} \quad$ cost of ballast in SEK $/ \mathrm{km}$

$C_{\mathrm{eb}} \quad$ equipment cost for ballast cleaning in SEK/h

$C_{\text {ebr }} \quad$ equipment cost for ballast renewal in SEK $/ \mathrm{h}$

$C_{\text {efr }} \quad$ equipment cost for fastener renewal in $\mathrm{SEK} / \mathrm{h}$

$C_{\mathrm{eg}} \quad$ equipment cost for grinding in $\mathrm{SEK} / \mathrm{h}$

$C_{\mathrm{er}} \quad$ equipment cost to repair rail breaks in SEK/h

$C_{\text {err }} \quad$ equipment cost for rail renewal in SEK/h

$C_{\text {esr }}$ equipment cost for sleeper renewal in SEK/h

$C_{\text {et }} \quad$ equipment cost for track inspection in SEK/h

$C_{\text {eta }} \quad$ equipment cost for tamping in SEK/h

$C_{\mathrm{f}} \quad$ cost of fasteners in SEK/ $\mathrm{km}$

$C_{\mathrm{L}} \quad$ average labour cost in Swedish Kroner (SEK) / $\mathrm{h}$

$C_{\mathrm{lu}} \quad$ cost of lubrication material for each lubricator per year in SEK 
$C_{\mathrm{r}} \quad$ cost of rail in SEK $/ \mathrm{km}$

$C_{\mathrm{s}} \quad$ cost of sleeper in SEK/ $\mathrm{km}$

$f_{\mathrm{rb}_{i}} \quad$ failure rate of rail (breaks) in the $i$ th curve

$K \quad$ class of curve radii

$L \quad$ total length of track section in $\mathrm{km}$

$L_{i} \quad$ length of $i$ th curve in $\mathrm{km}$

$L_{\mathrm{r}} \quad$ average length of rail replacement due to rail break

$m_{\mathrm{b}_{i}} \quad$ interval for ballast cleaning for $i$ th curve in MGT

$m_{\mathrm{br}_{i}} \quad$ interval for ballast renewal for $i$ th curve in MGT

$m_{\mathrm{fr}_{i}}$ interval for fastener renewal for $i$ th curve in MGT

$m_{\mathrm{g}_{i}}$

$m_{\mathrm{rb}_{i}}$

$m_{\mathrm{rr}_{i}}$

$m_{\mathrm{sr}_{i}}$ interval for grinding for $i$ th curve in MGT mean time to rail breaks in $i$ th curve in MGT interval for rail renewal for $i$ th curve in MGT

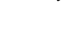

$m_{\mathrm{t}}$ interval for sleeper renewal for $i$ th curve in MGT

$m_{\mathrm{ta}_{i}}$ interval for track inspection in MGT interval for tamping for $i$ th curve in MGT
$M \quad$ gross tonnage per year in MGT

$M \quad$ life period of track in MGT

$n_{\mathrm{g}_{i}} \quad$ number of grinding passes on $i$ th curve

$n_{\mathrm{l}_{i}} \quad$ number of wayside lubricators in $i$ th curve

$N \quad$ life period of track (equivalent to $M$ ) in years

$r \quad$ discount rate

$T_{\mathrm{b}_{i}} \quad$ mean time to clean ballast for $i$ th curve in $\mathrm{h} / \mathrm{km}$

$T_{\mathrm{br}_{i}} \quad$ mean time for ballast renewal for $i$ th curve in $\mathrm{h} / \mathrm{km}$

$T_{\mathrm{fr}_{i}} \quad$ mean time for fastener renewal for $i$ th curve in $\mathrm{h} / \mathrm{km}$

$T_{\mathrm{g}_{i}} \quad$ mean time to grind for $i$ th curve in $\mathrm{h} / \mathrm{km}$

$T_{\text {lu }} \quad$ mean time to refill lubrication material for each lubricator in hour

$T_{\mathrm{rb}_{i}} \quad$ mean TTR rail break in $i$ th curve in hour

$T_{\operatorname{rr}_{i}} \quad$ mean time for rail renewal for $i$ th curve in $\mathrm{h} / \mathrm{km}$

$T_{\mathrm{sr}_{i}} \quad$ mean time for sleeper renewal for $i$ th curve in $\mathrm{h} / \mathrm{km}$

$T_{\mathrm{t}} \quad$ mean time to inspect track in $\mathrm{h} / \mathrm{km}$

$T_{\mathrm{ta}_{i}} \quad$ mean time to tamp for $i$ th curve in $\mathrm{h} / \mathrm{km}$ 